\author{
Stan C. Kwasny "* \\ The Ohio State University \\ Columbus, Ohio
}

I. Introduction

Among the components included in Natural Language Understanding (NLU) systems is a grammar which specifies much of the linguistic structure of the utterances that can be expected. However, it is certain that inputs that are 1ll-formed with respect to the grammar will be received, both because people regularly form ungrammatical utterances and because there are a variety of forms that cannot be readily included in current grammatical models and are hence "extra-grammatical". These might be rejected, but as Wilks stresses, "...understanding requires, at the very least, ... some attempt to interpret, rather than merely reject, what seem to be ill-formed utterances." [WIL76]

Th1s paper investigates several language phenomena commonly considered ungrammatical or extra-grammatical and proposes techniques directed at integrating them as much as possible into the conventional grammatical processing performed by NLU systems through Augmented Transition Network (ATN) grammars. For each NLU system, a "normative" grammar is assumed which specifies the structure of well-formed inputs. Rules that are both manually added to the original grammar or automatically constructed during parsing analyze the 11l-formed input. The 111-formedness is shown at the completion of a parse by deviance from fully grammatical structures. We have been able to do this processing while preserving the structural characteristics of the original grammar and its inherent efficiency.

Some of the phenomena discussed have been considered previously in particular NLU systems, see for example the ellipsis handing in LIFER [HEN77]. Some techniques similar to ours have been used for parsing, see for example the conjunction mechanism in LUNAR [W0073]. On the linguistic side. Chomsky [CHO64] and Katz [KAT64], among others have considered the treatment of ungrammaticality in Transformational Grammar theorles. The study closest to ours is that of Weischedel and Black [WEI79]. The present study is distinguished by the range of phenomena considered, its structural and efficiency goals, and the inclusion of the techniques proposed within one implementation.

This paper looks at these problems, proposes mechanisms aimed at solving the problems, and describes how these mechanisms are used. At the end, some extensions are suggested. Unless otherwise noted, all ideas have been tested through implementation. A more detailed and extended discussion of all points may be found in Kwasny [KWA79].

\section{Language Phenomena}

Success in handing ungrammatical and extra-grammatical input depends on two factors. The f1rst is the ldentification of types of 111-formedness and the patterns they follow. The second is the relating of 111-formed 1nput to the parsing path of a gramatical input the user intends. This section introduces the types of ill-formedness we have studied. * Current Address:

Computer Science Department

Indiana University

Blcomington, Ind lana
Norman K. Sondheimer

Sperry Univac

Blue Bell. Pennsylvania

and discusses their relationship to grammatical structures in terms of ATN grammars.

\section{I Co-Occurrence Violations}

Our first class of errors can be connected to co-occurrence restrictions within a sentence. There are many occassions in a sentence where two parts or more must agree (* indicates an 11l-formed or ungrammatical sentence):

"Draw a circles.

-I will stay from now under midnight.

The errors in the above involve coordination between the underlined words. The first example illustrates simple agreement problems. The second involves a complicated relation between at least the three underlined terms.

Such phenomena do occur naturally. For example, Shore [SHO77] analyzes fifty-six freshman English papers written by Black college students and reveals patterns of nonstandard usage ranging from uninflected plurals, possessives, and third person singulars to overinflection (use of inappropriate endings.)

For co-occurrence violations, the blocks that keep inputs from being parsed as the user intended arise from a failure of a test on an arc or the failure to satisfy an arc type restriction, e.g.. fallure of a word to be in the correct category. The essential block in the first example would likely occur on an agreement test on an arc accepting a noun. The essential blockage in the second example is likely to come from fallure of the arc testing the final preposition.

\section{II.2 Ellipsis and Extraneous Terms}

In handling ellipsis, the most relevant distinction to make $1 \mathrm{~s}$ between contextual and telegraphic ellipsis.

Contextual ellipsis occurs when a form only makes proper sense in the context of other sentences. For example, the form

* President Carter has.

seems ungrammatical without the preceding question form

Who has a daughter named Amy?

President Carter has.

Telegraphic ellipsis, on the other hand, occurs when a form only makes proper sense in a particular situation. For example, the forms

3 chairs no waiting (sign In barber shop)

Yanks split (headline in sports section)

Profit margins for each product

(query submitted to a NLU system) 
are cases of telegraphle ellipsis with the situation noted in parentheses. The rinal example is from an experimental study of NLU for management information which indicated that such forms must be considered [MAL75].

Another type of ungrammaticallty related to ellipsis occurs when the user puts unnecessary words or phrases in an utterance. The reason for an extra word may be a change of intention. In the middle of an utterance, an oversight, or simply for emphasis. For example.

\section{- Draw a line with from here to there.}

"List prices of single unit prices for 72 and 73.

The second example comes from Malhotra [MaL75].

The best way to see the errors in terms of the ATN is to think of the user as trying to complete a path through the grammar, but having produced an input that has too many or too few forms necessary to traverse all arcs.

\section{II.3 Conjunction}

Conjunction is an extremely common phenomenon, but it is seldon directly treated in a gramar. We have considered several types of conjunction. as in

simple forms of conjunction occur most frequently,

John Loves Mary and hates Sue.

Gapping occurs when internal segments of the second conjunct are olssing, as in

John loves Mary and Mary John.

The 11st form of conjunction occurs when more than two elements are joined in a single phrase, as in

John loves Mary, Sue, Nancy, and B111.

Correlative conjunction occurs in sentences to coordinate the joining of constituents, as in

John both loves and hates sue.

The reason conjuncts are generally loft out of gramars is that they can appear in so auny places that inclusion would dramatically increase the s12e of the grammar. The same argument appl les to the ungramatical phenomena. Since they allow so much variation compared to gramatical forms, Including them with existing techniques would dramatically increase the size of a grammar. Further there is a real distinction in terms of completeness and clarity of intent between grammatical and ungramatical forms. Hence we roel justifled in suggesting spectal techniques for their treotment.

\section{Proposed Mechanisms and How They Apply}

The following presentation of our techniques assumes an understanding of the ATN model. The technlques are applied to the language phenomena discussed in the previous section.

\section{I Relaxat1on Techniques}

The first two methods described are relaxation methods which allow the successful traversal of ATN arcs that might not otherwise be traversed. During parsing, whenever an arc cannot be taken, a check is made to see if some form of relaxation can apply. If it can, then a backtrack point is created which includes the rolaxed version of the are. These alternatives are not considered unt1l after all possible gramatical paths have been attempted thereby insuring that grammatical inputs are still handled correctly. Relaxation of previously relaxed arcs is also possibie. Two methods of relaxation have been investigated.

Our flrst method involves relaxing a test on an arc, similar to the method used by Wolschodel in [WEI79]. Test relaxation occurs when the test portion of an arc contains a relaxable predicate and the test fails. Two methods of test relaxation have been identified and implemented based on prodicate type. Predicates can be designated by the grammar writer as either absolutely violable in which case the opposite velue of the predicate (determined by the LISP function NoT applied to the predicate) is substituted for the predicate during relaxation or conditionally violable in which case a substitute predicate is provided. For exemple, consider the following to be a test that falls:

(AND

$$
\begin{aligned}
& \text { (INFLECTING V) } \\
& \text { (INTRANS V)) }
\end{aligned}
$$

If the prodicate INFLECIING was declered absolutely violable and its use in this test returned the value NIL, then the negation of (INFLECTING $V$ ) would replace it in the test creating now are with the test:

(AND

$$
\text { (INTRANS V)) }
$$

If INTRANS were conditionally violable with the substitute predicate TRANS, then the following test would appear on the new arc:

(AND

(INFLECTING $V$ )

(TRANS V))

Whonever more thon one tast in a falling are is violable, all possible single relaxations are attempted independently. Absolutely violable predicetes can be permitted in cases where the test describes some superflcial conslstency checking or where the test's failure or success doesn't have a dreot affect on meaning, while conditionaliy violeble predicates apply to predicates which must be relaxed cautiously or else loss of meaning may rosult.

Chomsky discusses the notion of organizing word categories hierarchically in developing his ideas on degroes of gramat1calness. We have applied and extended these 1deas in our second method of relaxation called category relaxation. In this method, the grammar writer produces, along with the gramar, a hierarchy describling the relationshlp among words, categories, and phrase types which is utilized by the relaxation meohanism to construct relaxed versions of arcs that have falled. When an are falls because of an are type fallure (1.0., because a particular word, category, or phrase was not found) a now arc (or arcs) may be created according to the description of the word, category, or phrase in the hierarchy. Typlaally. PUSH arcs will relax to PUSH ares. CAT ares to CAT or PUSH ares, and WRD or MEM aros to CAT arcs. Consider, for example, the syntactic category hlerarchy for pronouns shown in Figure 1. For this example, the category rolaxation 
mechanism would allow the relaxation of PERSONAL pronouns to include the category PRONOUN. The arc produced from category relaxation of PERSONAL pronouns also includes the subcategories REFLEXIVE and DEMONSTRATIVE in order to expand the scope of terms during relaxation. As with test relaxation, successive relaxations could occur.

For both methods of relaxation, "deviance notes" are generated which describe the nature of the relaxation in each case. Where multiple types or multiple levels of relaxation occur, a note is generated for each of these. The entire list of deviance notes accompanies the final structure produced by the parser. In this way, the final structure is marked as deviant and the nature of the deviance is available for use by other components of the understanding system.

In our implementation, test relaxation has been fully implemented, while category relaxation has been implemented for all cases except those involving PUSH arcs. Such an implementation is anticipated, but requires a modification to our backtracking algorithm.

\section{III.2 Co-Occurrence and Relaxation}

The solution being proposed to handled forms that are deviant because of co-occurrence violations centers around the use of relaxation methods. Where simple tests exist within a grammar to filter out unacceptable forms of the type noted above, these tests may be relaxed to allow the acceptance of these forms. This doesn't eliminate the need for such tests since these tests help in disambiguation and provide a means by which sentences are marked as having violated certain rules.

For co-occurrence violations, the point in the grammar where parsing becomes blocked is often exactly where the test or category violation occurs. An arc at that point is being attempted and fails due to a failure of the co-occurrence test or categorization requirements. Relaxation is then applied and an alternative generated which may be explored at a later point via backtracking. For example, the sentence:

* John Love Mary

shows a disagreement between the subject (John) and the verb (love). Most probably this would show up during parsing when an arc is attempted which is expecting the verb of the sentence. The test would fall and the traversal would not be allowed. At that point, an ungrammatical alternative is created for later backtracking to consider.

\section{III.3 Patterns and the Pattern Arc}

In thls section, relaxation techniques, as applied to the grammar itself, are introduced through the use of patterns and pattern-matching algorithms. Other systems have used patterns for parsing. We have devised a powerful method of integrating, within the ATN formalism, patterns which are rlexible and useful.

In our current formulation, which we have implemented and are now testing, a pattern is a linear sequence of ATN arcs which is matched against the input string. A pattern arc (PAT) has been added to the ATN formalism whose form is similar to that of other arcs:

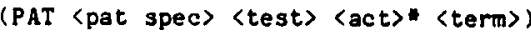

The pattern specification (<pat spec〉) is defined as:

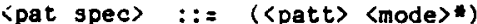

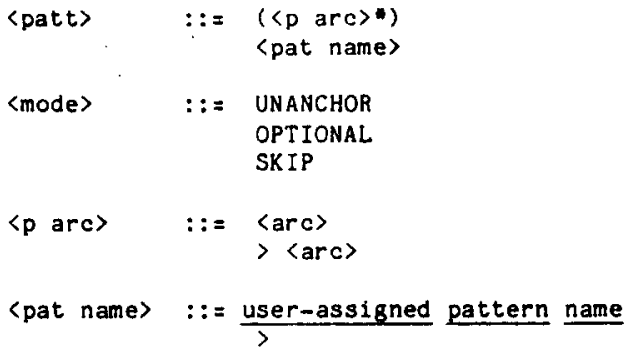

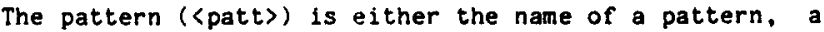
">", or a list of ATN arcs, each of which may be preceded by the symbol ">", while the pattern mode (〈mode〉) can be any of the keywords, UNANCHOR, OPTIONAL, or SKIP. These are discussed below. To refer to patterns by name, a dictionary of patterns is supported. A dictionary of arcs is also supported, allowing the referencing of aros by name as well. Further, named arcs are defined as macros, allowing the dictionary and the grammar to be substantially reduced in size.

\section{THE PATTERN MATCHER}

Pattern matching proceeds by matching each arc in the pattern against the input string, but is affected by the chosen "mode" of matching. Since the individual component arcs are, in a sense, complex patterns, the ATN interpreter can be considered part of the matching algorithm as well. In arcs within patterns, explicit transfer to a new state is ignored and the next arc attempted on success is the one following in the pattern. An arc in a pattern prefaced by ">" can be considered optional, if the OPTIONAL mode has been selected to activate this feature. When this is done, the matching algorithm still attempts to match optional arcs, but may ignore them. A pattern unanchoring capability is activated by specifying the mode UNANCHOR. In this mode, patterns are permitted to skip words prior to matching. Finally, selection of the SKIP mode results in words being ignored between matches of the arcs within a pattern. This is a generalization of the UNANCHOR mode.

Pattern matching again results in deviance notes. For patterns, they contain information necessary to determine how matching succeeded.

\section{SOURCE OF PATTERNS}

An automatic pattern generation mechanism has been implemented using the trace of the current execution path to produce a pattern. This is invoked by using a ">" as the pattern name. Patterns produced in this fashion contain only those arcs traversed at the current level of recursion in the network, although we are planning to implement a generalization of this in which PUSH arcs can be automatically replaced by their subnetwork paths. Each arc in an automatic pattern 13 marked as optional. Patterns can also be constructed dynamically in precisely the same way grammatical structures are built using BUILDQ. The vehicle by which this is accomplished is discussed next.

\section{AUTOMATIC PRODUCTION OF ARCS}

Pattern arcs enter the grammer in two ways. They are manually written into the grammar in those cases where the ungrammaticalities are common and they are added to the grammar automatically in those cases where the ungrammaticality is dependent on context. Pattern arcs produced dynamically enter the grammar through one of two devices. They may be constructed as needed by 
special macro arcs or they may be constructed for future use through an expectation mechanism.

As the expectation-based parsing efforts clearly show. syntactic elements especially words contain important clues on processing. Indeed, we also have found it useful to make the ATN mechanism more "active" by allowing it to produce new arcs based on such clues. To achleve this, the CAT. MEM, TST, and WRD arcs have been generalized and four new "macro" arcs, known as CAT*, MEM*, TST*, and WRD*, have been added to the ATN formalism. These are similar in every way to their counterparts, except that as a final action, instead of indicating the state to which the traversal ieads, a new arc is constructed dynamically and immediately executed. The difference in the form that the new are takes is seen in the following pair where 〈creat act) is used to define the dynamic are:

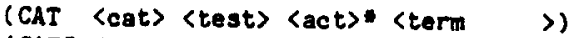

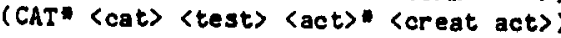

Arcs computed by macro arcs can be of any type permitted by the ATN, but one of the most useful arcs to compute in this manner is the PAT arc discussed above.

\section{EXPECTATIONS}

The macro arc forces immediate execution of an aro. Arcs may also be computed and temporarily added to the grammar for later execution through on "expectation" mechanism. Expectations are performed as actions within arcs (analogous to the HOLD action for parsing structures) or as actions elsewhere in the MLU system (e.8.. during generation when particular types of responses can be foreseen). Two forms are allowed:

(EXPECT 〈creat act〉 〈state〉)

(EXPECT 〈creat act>

In the first case, the arc created is bound to a state as specifled. When later processing leads to that state, the expected arc will be attempted as one alternative at that state. In the second case, where no state is specified, the effect is to attempt the arc at every state visited during the parse.

The range of an expectation produced during parsing is ordinarily Iimlted to a single sentence, with the arc d1sappearing after it has been used; however, the start state, S*, is reserved for expectations intended to be active at the beginning of the next sentence. These will disappear in turn at the end of processing for that sentence.

\section{III.4 Patterns, El11psis, and Extraneous Forms}

The Pattern arc is proposed as the primary mechanism for handling ellipsis and extraneous forms. A Pattern arc can be seen as capturing a single path through a network. The matcher gives some freedow in how that path relates to a string. We propose that the appropriate parsing path through a network relates to an elliptical sentence or one with extra words in the same way. With contextual ellipsis, the relationship will be in having some of the arcs on the correct path not satisfied. In pattern arcs, these will be represented by arcs marked as optional. With contextual ellipsis. dialogue context will provide the defaults for the missing components. With Pattern arcs, the deviance notes will show what was left out and the other components in the MUU system will be responsible for supplying the values.
The source of patterns for contextual ellipsis is important. In Lifer [HEN77], the prevlous user input can be seen as a pattern for elliptical processing of the current input. The automatic pattern generator developed here, along with the expectation mechanism, will capture this level of processing. But with the ability to construct arbitrary patterns and to add then to the grammar from other components of the NLU system, our approach can accoomplish much more. For example, a question Beneration routine could add an expectation of a yes/no answer in front of a transformed rephrasing of a question, as in

\section{Did Amy klss anyone?}

Yes, Jimmy was k1ssed.

Patterns for telegraphic ellipsis will have to be added to the grammar manually. Generally, patterns of usage must be ldentifled, say in a study like that of Malhotra, so that appropriate patterns can be constructed. Patterns for extraneous forms w11l also be added in advance. These will either use the unachor option in order to skip false starts, or dynamically produced patterns to catch repetitions for emphasis. In general, only a $11 \mathrm{mited}$ number of these patterns should be required. The value of the pattern mechanism here. especially in the case of telegraphic ellipsis, w1ll be in connecting the ungramatical to grammatical forms.

\section{III.5 Conjunction and Macro Arcs}

Pattern arcs are also proposed as the primary mechenlsw for handling conjunction. The rationale for this is the often noted connection between conjunction and ellipsis, see for example Halliday and Hasan [HAL76]. Th1s is clear with Bapping, as in the following where the parentheses show the missing component

John Loves Mary and Mary (loves) John.

But it also can be seen with other forms, as in

John loves Mary and (John) hates Sue.

John Loves Mary. (John loves) Sue, (John loves) Nancy, and (John loves) Bill.

Whenever a conjunction is seen, a pattern is developed from the already identified elements and watched against the remaining segments of input. The heuristics for deciding from which level to produce the pattern force the most general interpretation in order to encourage an -111ptical reading.

All of the forms of conjunction described above are treated through a globally defined set of "confunction aros" (Some restricted cases, such as "and" following "between", have the conjunction built into the grammar). In general. this set will be made up of macro arcs which compute Pattern arcs. The automatic pattern mechanism is heavily used. With simple conjunctions, the rightmost elements in the patterns are matched. Internal elements in patterns are skipped with gapping. The 11st form of conjunction can also be handled through the careful construction of dynamic patterns which aro then expected at a later point. Correlatives are treated similarly, with expectations based on the dynanic bullding of patterns.

There are number of details in our proposal which w11 not be presented. There are also visible ilmits. It is Instructive to compere the proposal to the SYSCONJ facility of Woods [W0073]. It treats conjunction as 
showing alternative ways of continuing a sentence. This allows for sentences such as

He drove his car through and broke a plat..? glass window.

which at best we will accept with a mislsading deviance note. However, it can not handle the obvious elliptical cases, such gapping, or the tightly constrained cases, such as correlatives. We expect to continue investigating the pattern approach.

\section{III.6 Interaction of Techniques}

As grammatical processing proceeds, ungrammatical possibilities are continually being suggested from the various mechanisms we have implemented. To coordinate all of these activitles, the backtracking mechanism has been improved to keep track of these alternatives. AII paths in the original grammar are attempted first. Only when these all fall are the conjunction alternatives and the manually added and dynamically produced ungrammatical alternatives tried. All of the alternatives of these sorts connected with a single state can be thought of as a single possibility. A selection mechanism is used to determine which backtrack point among the many potential alternatives is worth exploring next. Currently, we use a method also used by Weischedel and Black [WEI79] of selecting the alternative with the longest path length.

\section{Conclusion and Open Questions}

These results are significant, we believe, because they extend the state of the art in several ways. Most obvious are the following:

The use of the category hierarchy to handle arc type fallures;

The use of the pattern mechanism to allow for contextual ellipsis and gapping;

More generally, the use of patterns to allow for many sorts of ellipsis and conjunctions; and

Finally, the orchestration of all of the techniques in one coherent system, where because all grammatical alternatives are tried rirst and no modifications are made to the original grammar, 1ts inherent efflciency and structure are preserved.

\section{IV.1 Open Problems}

Various questions for further research have arisen during the course of this work. The most important of these are discussed here.

Better control must be exercised over the selection of viable alternatives when ungrammatical possibilities are being attempted. The longest-path heuristic is somewhat weak. The process that decides this would need to take into consideration, among other things, whether to allow relaxation of a criteria applied to the subject or to the verb in a case where the subject and verb do not agree. The current path length heuristic would always relax the verb whlch is clearly not always correct.

No consideration has been given to the possible connection of one error with another. In some cases, one error can lead to or affect another.
Several other types of ill-formedness have not been considered in this study, for example, idioms, metaphors, incorrect word order, run together sentences. incorrect punctuation, misspelling, and presuppositional fallure. Either little is known about these processes or they have been studied elsewhere independently. In either case, work remains to be done.

\section{Acknowledgments}

We wish to acknowledge the comments of Ralph Weischedel and Marc Fogel on previous drafts of this paper. Although we would like to blame them, any shortcomings are clearly our own fault.

\section{Bibliography}

[CH064] Chomsky, N.. "Degrees of Grammaticalness," in [FOD64], 384-389.

[FOD64] Fodor, J.A. and J, J. Katz. The Structure of Language: Readings in the Philosophy of Language, Prentice-Hall, Englewood Cliffs, New Jersey, 1964.

[HAL76] Halliday, M.A.K, and R. Hasan, Cohesion in English, Longman, London, 1976.

[HEN77] Hendrix, G. G., "The LIFER Manual," Technical Note 138, Artificial Inteligence Center. Stanford Research Institute, Menlo Park. California, February, 1977

[KAT64] Katz, J.J., "Semi-Sentences," in [FOD64], 400-416.

[KWA79] Kwasny, S., "Treatment of Ungrammatical and Extragrammatical Phenomena in Natural Language Understanding Systems," PhD dissertation (forthooming), Ohio State University, 1979.

[MAL75] Malhotra, A., "Design Criteria for a Knowledge-Based English Language System for Management: An Experimental Analysis." MAC TR-146, M.I.T., Cambridge, Ma, February, 1975.

[SH077] Shores, D. L., "Black English and Black Attitudes," in Papers in Language Variation. D. L. Shores and C. P. Hines (Ed.). The University of Alabama Press, University. Alabama, 1977.

[WEI79] Weischedel, R. M., and J. Black, "Responding to Potentially Unparseable Sentences," manuscript. Department of Computer and Information Sclences, University of Delaware, Newark, Delaware, 1979.

[WIL76] Wilks, Y.. "Natural Language Understanding Systems Within the A.I. Paradigm: A Survey," American Journal of Computational Linguistics. microflche $40,1,1976$

[W0073] Woods, W. A: "An Experimental Parsing system for Transition Network Gramars," In Natural Language Processing, R. Rustin (Ed.). Algorithnics Press, 1973.

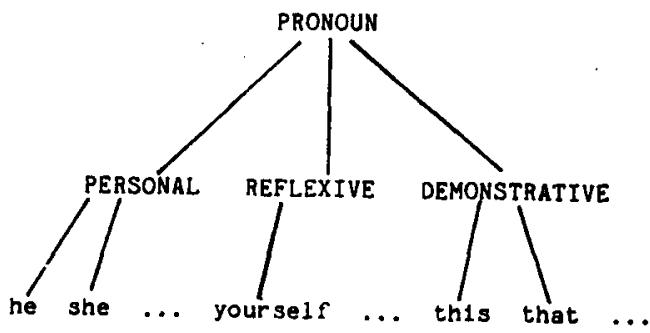

Figure 1. A Category Hierarchy 
